\title{
128 halftone patterns for printing gray-level images on a laser printer
}

\author{
MIGUEL A. GARCÍA-PÉREZ \\ Universidad Complutense, Madrid, Spain
}

\begin{abstract}
This paper presents a set of 128 halftone patterns on an $8 \times 8$ block size. The patterns have been designed to produce an acceptable gray-scale rendition when the halftone is seen from a distance and, at the same time, to allow comparisons to be made among the intensity values of individual pixels at a closer look. A Pascal program is also presented that produces halftones on an HP Laserjet+ laser printer, and sample halftones are shown. Finally, a method is proposed that prevents images digitized with a $4: 3$ aspect ratio from being distorted when halftones are printed at the usual 1:1 aspect ratio.
\end{abstract}

Years ago, the display of continuous-tone images with digital computers was a challenge that stimulated engineers' imaginations (see Knowlton \& Harmon, 1972, for some initial proposals). The problem that had to be solved was that of producing a gray-scale rendition on a bilevel display - a display whose cells or elements can only be in one of two states, either lit or unlit. Presently, a variety of graphic systems that can be easily interfaced to digital computers allow a number of gray levels to be directly displayed on a cathode-ray tube by varying the intensity of each picture element (pixel). As many as eight bits (one byte) are used by the smaller systems to store and display information about every pixel, thus yielding a range of $2^{8}=256$ gray levels per pixel.

This technology has been extremely useful in the development of image-processing algorithms, making possible the immediate visualization of output images. In all research areas where image processing is used as a tool, ranging from computer vision to the modeling of human visual processing, images that are to be processed and their corresponding outputs are rarely larger than $512 \times 512$ pixels. When the results of such research are to be printed, the images on the face of the monitor are not in a suitable format. The conventional approach to having images printed is to take snapshots of the displayed images. This process is somewhat expensive, and almost anyone who has done it knows its drawbacks well: contrast is affected in a way that is difficult to control during the photographic process, the images appear distorted due to the geometry of photographic lenses and to the nonplanar contour of the monitor screen, and so forth.

An alternative to photographing the images on the monitor is to print them directly on a standard printer with graphics capability. Although almost any printer meet-

This work has been supported with grants from the Universidad Complutense and the Fundación Ramón Areces for a research project entitled Visión Artificial y Visión Humana: Aplicaciones a la Percepción Visual en Robótica. Address correspondence to Miguel A. García-Pérez, Laboratorio de Visión, Facultad de Psicología, Universidad Complutense, Campus de Somosaguas, 28023 Madrid, Spain. ing this requirement will serve, laser printers are preferable over dot-matrix printers because of their better print resolution and their accuracy of positioning control. Also, unlike dot-matrix printers, laser printers do not suffer from ribbon wear. Nevertheless, laser printers are presently bilevel devices and printing gray-level images on them requires halftoning techniques. Halftoning has been recently preferred over taking photographs by several authors (e.g., Förstner, 1987, Figure 1; Germani, Jetto, \& Verdicchio, 1986, Figure 4; Zucker, 1986, Figure 1).

In general, halftones are produced by assigning either a single display element or several to every pixel in the digital image. In both cases, a halftoning algorithm controls whether each of the display elements will be black or white at print time. The first method will not be considered here since the conventional size of digital images will result in very small halftones when printed at the conventional laser printer resolution of $\mathbf{3 0 0}$ dots per in. (dpi). (A useful algorithm of this kind, as well as references to others, can be found in Anastassiou \& Pennington, 1982.) When the second method is used, the display elements allocated to every pixel are usually arranged in a square or rectangular shape which is called a halfione block. Halftone blocks are used to define halftone patterns by setting every element in the block to black or white differently. Every pixel in the digital image is then mapped onto the halftone pattern that corresponds to its gray level, and patterns are printed instead of pixels to produce the halftone image.

It has been suggested (Anastassiou \& Pennington, 1982; Newman \& Sproull, 1981, p. 225) that if a block of $n$ elements is used, then $n+1$ gray levels can be producedthat is, as many patterns as can be generated by varying the number of black elements from 0 to $n$. Chen, Mintzer, and Pennington (1987), following this approach, designed the patterns to achieve the largest compression efficiency for transmission of halftone images. Contrary to the belief that large blocks must be used if a wide range of gray levels is needed, this paper describes a set of 128 
halftone patterns formed in $8 \times 8$ halftone blocks (i.e., with $n=64$ ). Some of these patterns have identical numbers of black and white elements, but their different arrangement within the block makes the perceived darkness of each pattern different. Simplistic considerations ordinarily lead us to conclude that the objective reflectivity of a pattern depends solely on the relative areas of black and white elements in the block and, hence, that the number of different gray levels that can be achieved is $n+1$. However, such reasoning is not valid when human inspection of halftones is concerned, since our visual system has both spatial integration areas that do not match the halftone-block size and functional properties of contrast enhancement and assimilation (White, 1982) that make perceived brightness different from objective intensity.

However, designing more than $n+1$ patterns was not the main goal of this development. In the modeling of human spatial vision, filtering has been a common method of showing the consequences of visual processing. For example, Ginsburg (1986) has reported results regarding the Hermann illusion, which showed that the illusory spots might be explained as a result of the band-pass filtering performed by the human visual system. His argument was that the gray spots actually appeared in the filtered image. Inspection of that image reveals this fact, but it is not clear whether the spots are physically present in the image or actually illusory, added by our visual system when we look at the filtered image just as is the case when inspecting the original, unfiltered image. To resolve the question in favor of the former interpretation, he provided a numerical printout of the intensity values at every pixel in the filtered image, thus showing that the intensity at the intersection of streets was actually darker. The halftone patterns presented in this paper have been expressly designed not only to give a perceptually pleasing graylevel rendition when seen from a distance, but also to provide at the same time objective clues to the relative image intensities at the pixels when closely looked at, and thus eliminate the need to resort to printing pixel values as Ginsburg (1986) was forced to do.

The halftone patterns and their underlying design philosophy are described in the next section, where some halftone images are also shown. Then, a Pascal program to produce halftones on an HP Laserjet + laser printer using these patterns is presented and its operation briefly explained. Next, a procedure to cope with differences between the aspect ratios of digitizers and laser printers is presented, which will prevent printed halftones from being distorted as compared with the gray-level images that were processed to produce them.

\section{Halftone Patterns}

The 128 halftone patterns are shown (enlarged) in Figure 1. From top to bottom (left to right within each row), each pattern corresponds to a gray level in the digital image from 0 (black) to 127 (white). Grid lines are used to help see the block and element boundaries, but they are not part of the patterns. Two main features of these patterns are important. First, since one of our goals is to provide clues to the relative intensities of the pixels, each printed pattern in the halftone must be kept as separately from its neighbors as possible so that it can be independently inspected and compared with them at a close distance. Hence, patterns were created by starting from the all white block and subsequently, with black dots, filling in more and more elements near the center of the block. Second, this general rule was violated from time to time to produce a different arrangement of the same number of black dots, so that its perceived brightness would result in an intermediate level between those corresponding to patterns varying only in the number of black dots. The particular set of patterns that were finally chosen, as well as the mapping of gray levels in the digital image onto them, was decided by judging the subjective ramp-like appearance of a gradient image such as that shown in Figure 2. This approach is similar to that followed by designers of digital type when adapting existing type styles to laser printers for use with personal computer desktop publishing: to make letters look smooth and consistent, they often trick the eye by carefully adding dots where they do not belong.

Figure 2 shows a 300 dpi printout of a halftone of the luminance gradient

$$
m(i, j)=i, \text { where } i, j=0,1, \ldots, 127 .
$$

As can be seen, the gradient rendering is perceptually good. Figure 3 shows a Gabor function of the type that is usually taken as a model of the receptive field of simple cells in the visual cortex. This picture shows that the patterns in Figure 1 perform well at producing slowly varying intensity changes. Figures 2 and 3 have both been produced on an HP Laserjet + laser printer by means of a simple program that is briefly described in the next section.

\section{Halftoning Program}

To produce the halftone of a digital image with the patterns in Figure 1, one merely has to read the file where

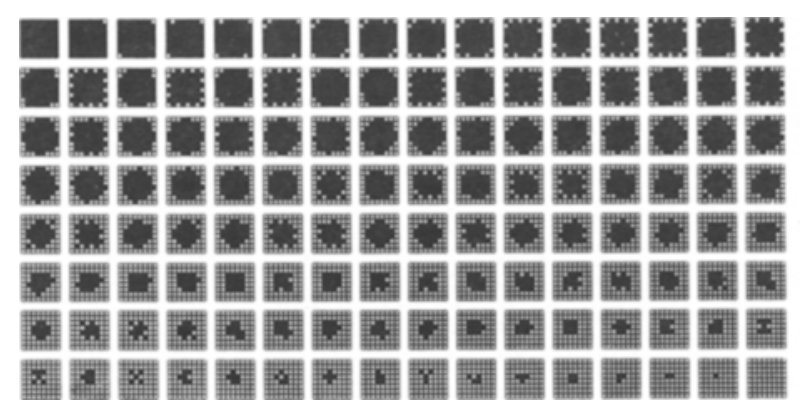

Figure 1. Halftone patterns. The (all black) pattern in the upper left corner corresponds to Gray Level 0 and the (all white) pottern in the lower right corner corresponds to Gray Level 127. The patterns are arranged so that the corresponding gray-level increases left to right from the topmost to the bottommost row. 


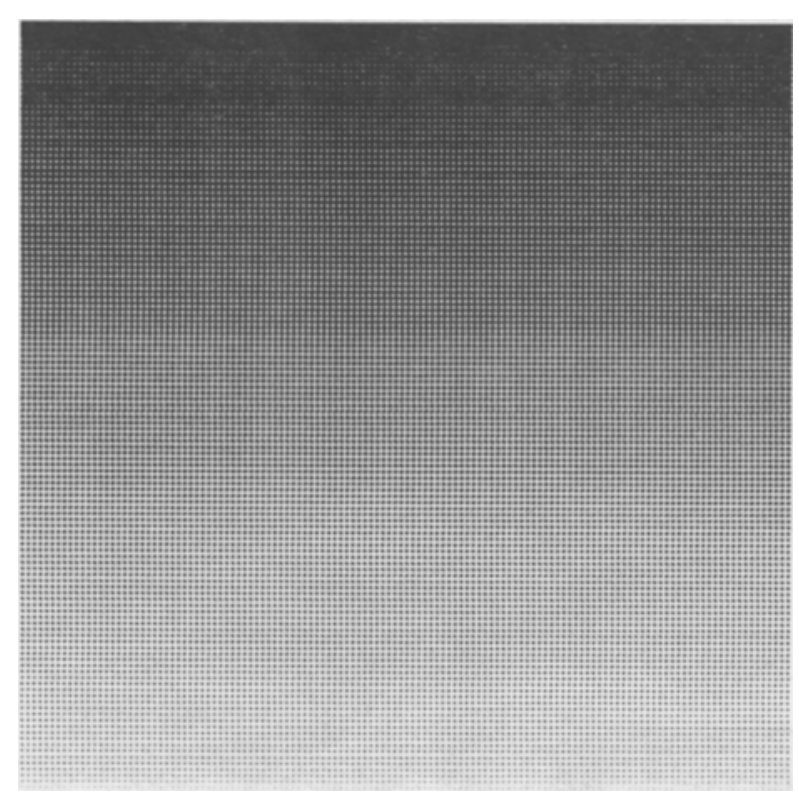

Figure 2. Luminance gradient image showing the gray scale that is produced with the halftone patterns in Figure 1.

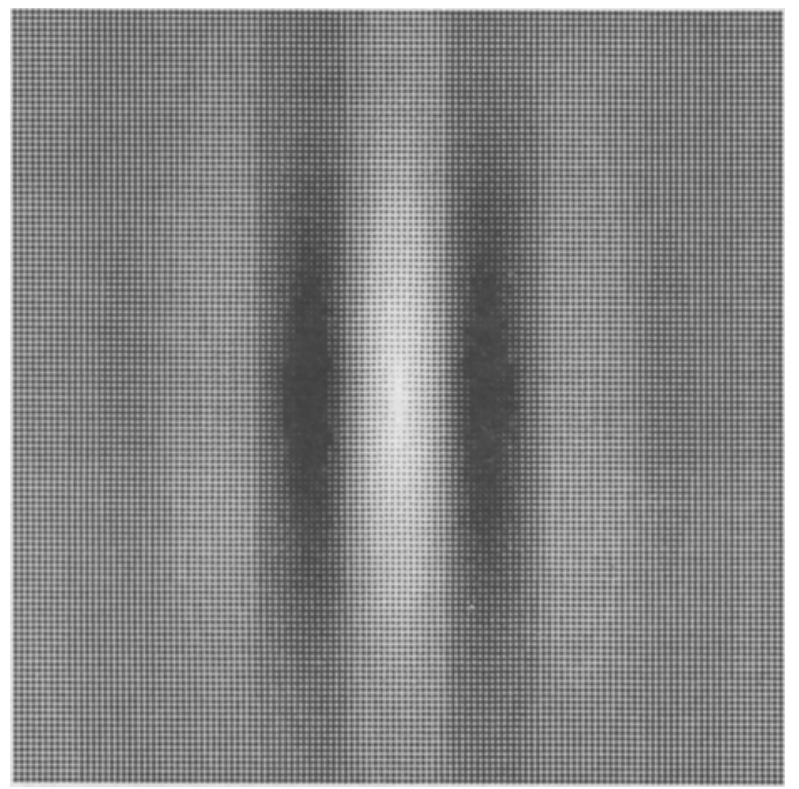

Figure 3. Gabor function describing a simple cell's receptive field model produced with the halftone patterns in Figure 1.

the image is stored, and draw, in position, the pattern corresponding to the gray level of each pixel in the image. This simple process requires some steps in order for the halftone to be printed. An initial step is to code the patterns so that the printer can print them easily. In the graphics mode, the atom that the Laserjet + prints is a row of eight dots (either white or black). This means that each pattern can be printed in eight consecutive rows vertically aligned in a column. To print a row, an ASCII character must be sent to the printer, which is determined as follows. The desired sequence of eight black or white dots is translated into a sequence of eight $1 \mathrm{~s}$ or $0 \mathrm{~s}$, with $1 \mathrm{~s}$ standing for black dots and 0s for white dots. This sequence is then regarded as an eight-bit binary number with the leftmost bit being the most significant. Finally, this binary number is converted to its decimal equivalent, which is the ASCII code of the character to be sent (see Hewlett Packard, 1986, pp. 2-40 to 2-42).

This process is illustrated in Figure 4 for the 48th pattern in Figure 1. This pattern is, then coded as a column of eight decimal numbers (ASCII codes). Instead of coding each pattern as needed during printing, it is more efficient first to code all patterns by writing them to a file and then to read the file at print time, storing codes in an $8 \times 128$ array.

Appendix A shows a Pascal program that creates halftones of $128 \times 128$ images. After some initialization statements, the program performs the following steps:

Step 1: read the image into an array.

Step 2: read the pattern codes into a second array.

Step 3: rescale the image values linearly to fit the range from 0 to 127 .

Step 4: initialize printer.

Step 5: for each row in the image array, from the topmost to the bottommost pattern code, send the ASCII character corresponding to every pixel in that row.

The third step is useful for enhancing contrast in the image and preventing 8-bit images from causing the program to search for a halftone pattern out of range. Patterns are not printed on a pixel by pixel basis in Step 5, for the sake of efficiency and memory-use optimization.

\section{Variations in Aspect Ratio}

The images presented thus far have been digitally synthesized. In this process, the same scaling factors are usually applied to both rows and columns, and the images are said to be produced at a 1:1 aspect ratio. This aspect ratio matches perfectly with the Laserjet + resolution, which is equal both vertically and horizontally. Nonetheless, frame grabbers used to digitize video signals usually yield images with a $4: 3$ aspect ratio. This is because the same numbers of samples are taken both vertically and horizontally, whereas video images are one

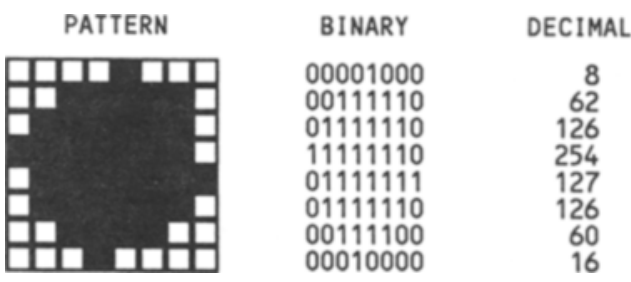

Figure 4. Coding of halftone patterns for efficient printing on an HP Laserjet+. See text for details. 
and one third times as wide as they are tall. Its only consequence is that images are not square-shaped when displayed on a graphics system, as shown in Figure 5 (top) for a $128 \times 128$ image. If these images are halftoned at a 1:1 aspect ratio, the result will be that the picture appears distorted as compared with its original counterpart, as if it had been vertically enlarged or horizontally compressed to leave it square-shaped (see Figure 5, center).

To handle this problem, the distorted halftone can be postprocessed, applying a vertical minification or horizontal magnification process by deriving one-dimensional versions of the two-dimensional algorithms described by Hou (1987). A simpler alternative is to counteract the distortion before halftoning, by distorting the image to the same extent, but in a direction opposite to the change from acquisition to printing aspect ratio. A fast and easy way of doing this is to apply Burt's (1981) one-dimensional, reduced, fractional, hierarchical discrete correlation (HDC) with order $4 / 3$ to every column in the image array. The algorithm is described in detail in Appendix B, and, when applied to a $128 \times 128$ image, it produces a $96 \times 128$ image that gets halftoned as shown in Figure 5 (bottom). The bottom image in Figure 5 appears somewhat more blurred than that in the center figure, because the HDC is a form of low-pass filtering. This effect can be counteracted by applying Laplacian edge enhancement, as described by Goertzel \& Thompson (1987), to the output image from HDC.

\section{Concluding Remarks}

In the halftone patterns presented in this paper, high spatial frequency rendition has been traded off for pixel identifiability. In this regard, vertical/horizontal alignment of clusters of dots has been preferred over the diagonal alignment that is more pleasing to our visual system; and 128 patterns have been designed to achieve an appropriate gray-scale rendition under these circumstances.

Other halftoning schemes exist, referred to earlier, that render more pleasing results. The new feature of the patterns presented in this paper is that they provide an acceptable rendition of gray levels when the halftone is viewed from a distance, while the relative intensities of individual pixels can be judged from a closer view.

Halftones are easily produced with the program presented here, and their perceived quality is much better than that of halftones produced by several investigators in image processing and visual modeling (Förstner, 1987; Germani et al., 1986; Zucker, 1986). This program can be readily adapted to print halftones on a different laser printer, and it is not restricted to printing as small as $128 \times 128$ images, provided that the printer to be used has enough memory to store the resulting halftone.

\section{Availability}

The Pascal program and pattern-code files are available from the author through electronic mail upon request. The author's address is W380@EMDUCM11(EARN).
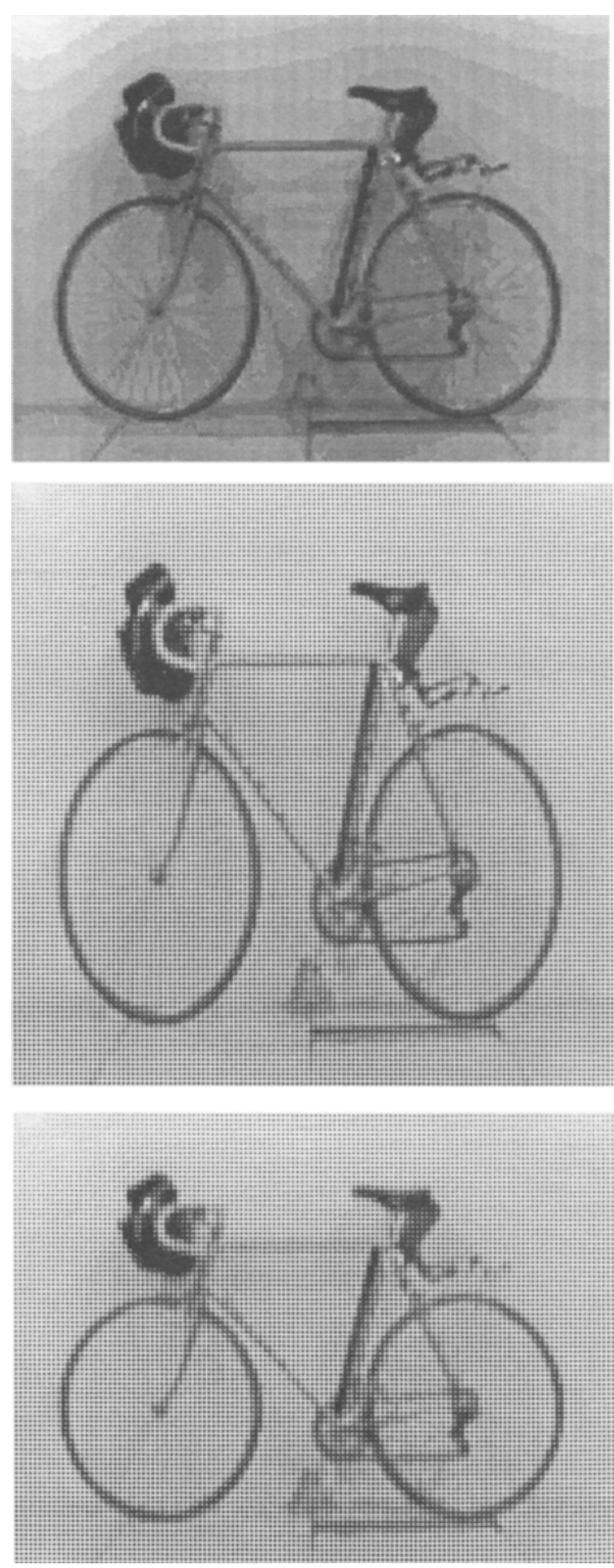

Figure 5. (Top) Image of a bicycle as displayed on the graphic system with which it was digitized from a video signal with $4: 3$ aspect ratio. (Center) Halftone image produced with 1:1 aspect ratio. Note distortions due to variations in aspect ratio. (Bottom) Halfone after preprocessing the digital image so as to compensate for distortions produced by aspect ratio variations. 


\section{REFERENCES}

Anastassiou, D., \& Pennington, K. S. (1982). Digital halftoning of images. IBM Journal of Research \& Development, 26, 687-697.

BURT, P. J. (1981). Fast filter transforms for image processing. Computer Graphics \& Image Processing, 16, 20-51.

Chen, Y.-H., Mintzer, F. C., \& Pennington, K. S. (1987). PANDA: Processing algorithm for noncoded document arquisition. IBM Journal of Research \& Development, 31, 32-43

FÖRSTNER, W. (1987). Reliability analysis of parameter estimation in linear models with applications to mensuration problems in computer vision. Computer Vision, Graphics, \& Image Processing, 40, 273-310.

GarcíA-P'́rez, M. A. (1988). HDC: A subroutine library for image processing using Burt's hierarchical discrete correlation. Behavior Research Methods, Instruments, \& Computers, 20, 317-329.

Germani, A., Jetto, L., \& Verdicchio, G. (1986). A bidimensional Kalman filtering approach to noisy image restoration. In V. Cappellini \& R. Marconi (Eds.), Advances in image processing and pattern recognition (pp. 75-82). Amsterdam: Elsevier.
Ginsburg, A. P. (1986). Spatial filtering and visual form perception. In K. R. Boff, L. Kaufman, \& J. P. Thomas (Eds.), Handbook of perception and human performance: Vol. II. Cognitive processes and performance (chap. 34). New York, NY: Wiley.

GoerTzel, G., \& Thompson, G. R. (1987). Digital halftoning on the IBM 4250 printer. IBM Journal of Research \& Development, 31, 2-15.

Hewlett-PaCKard. (1986). Laserjet Printer Family Technical Reference Manual. Palo Alto, CA: Author.

Hou, H. S. (1987). Digital processing of low resolution halftone images. Optical Engineering, 26, 603-608.

Knowlton, K., \& Harmon, L. (1972). Computer produced grey scales. Computer Graphics \& Image Processing, 1, 1-20.

Newman, W. M., \& Sproull, R. F. (1981). Principles of interact:ve computer graphics (2nd ed.). New York: McGraw-Hill.

WHITE, M. (1982). The assimilation-enhancing effect of a dotted surround upon a dotted test region. Perception, 11, 103-106.

ZUCKER, S. W. (1986). The computational connection in vision: Early orientation selection. Behavior Research Methods, Instruments, \& Computers, 18, 608-617.

APPENDIX A

Pascal Program to Produce Halftones

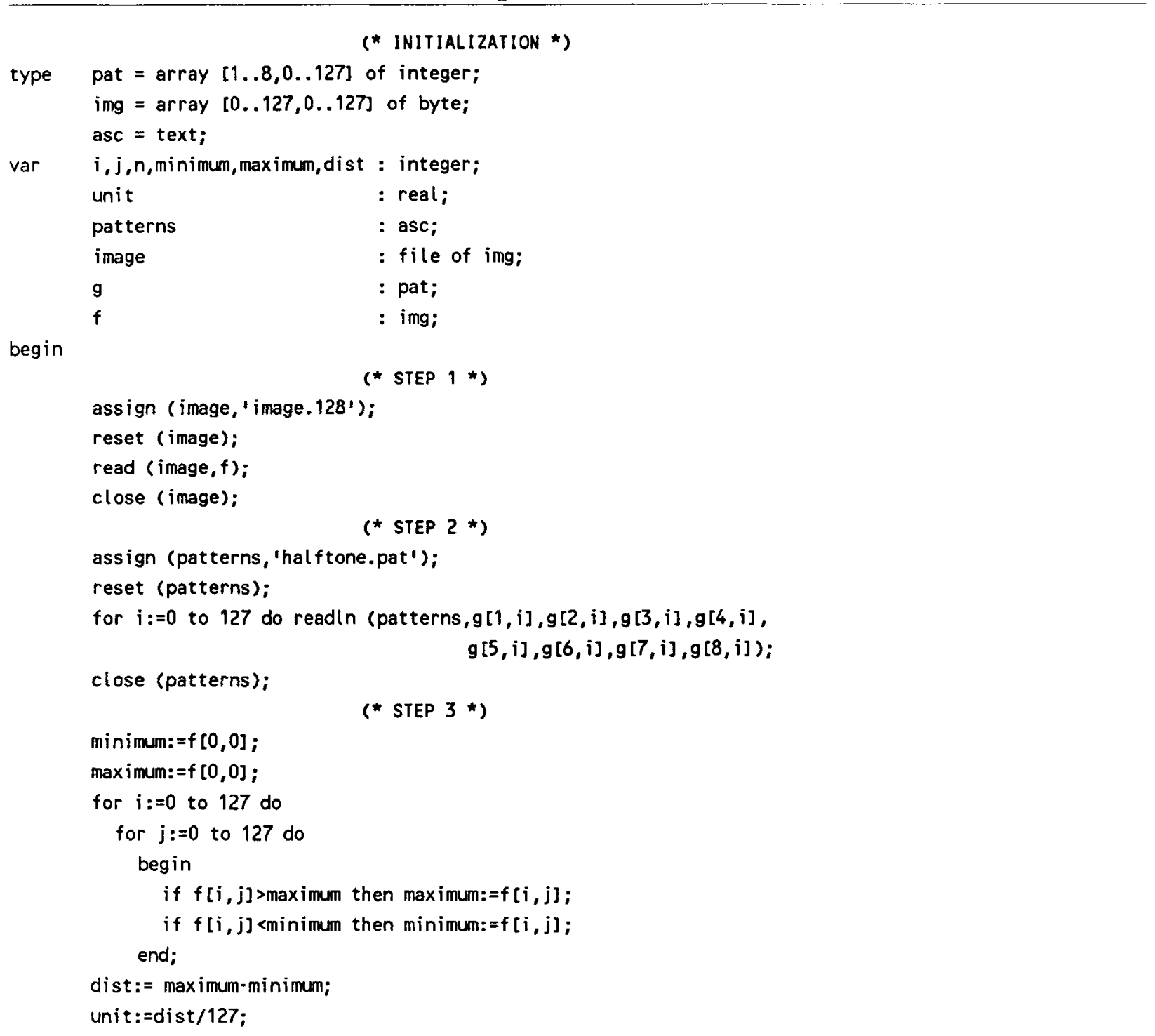




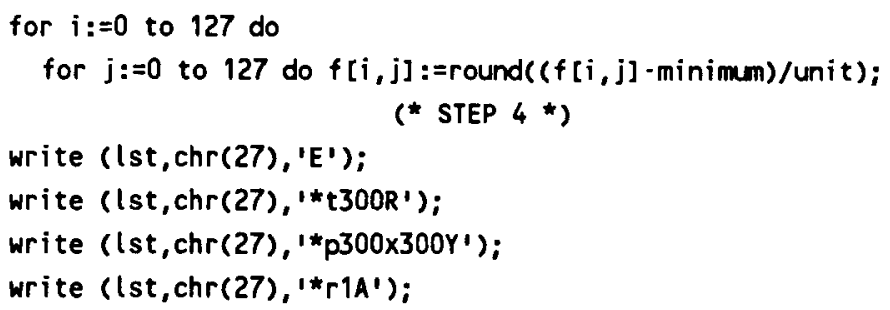

\author{
(* RESET PRIHTER *) \\ (* SET 300 DPI RESOLUTION *) \\ (* SET PRINT ORIGIN *) \\ (* START RASTER GRAPHICS *)
}

(* FOR EACH ROW IN THE IMAGE *)

(* FROM THE UPMOST TO THE BOTTOMMOST PATTERN COOE *)
(* FOR ALL COLUMNS IN THE IMAGE *)

(* 128 BYTES ARE TO BE SENT *)

\author{
(* END RASTER GRAPHICS *) \\ (* RESET PRINTER AND EJECT PAGE *)
}

end.

\begin{abstract}
APPENDIX B
Algorithm for Fractional HDC with Order $r=4 / 3$

Fractional HDC has been discussed by Burt (1981) and exemplified for order $r=3 / 2$ in that paper. There is no general form of the algorithm, and each fractional order requires particular consideration. The algorithm for one-dimensional HDC with fractional order $r=k_{1} / k_{2}=4 / 3$ is presented next. The derivation assumes that the number of input samples $n$ is a multiple of $k_{1}$. For general background on HDC, the reader is referred to Burt (1981) and García-Pérez (1988).

In its reduced form, fractional HDC produces $k_{2}$ output samples for every $k_{1}$ sample in the input sequence. When $r=4 / 3$, there are 96 output pixels for a 128-pixel input sequence. The resulting sequence is a low-pass filtered version of the original, since the value at each output sample is computed as a weighted average of the input samples in its immediate neighborhood. To specify the algorithm completely, it is necessary to make a choice as to the extent of this neighborhood. It is also necessary to de-
\end{abstract}

cide how the output sequence will be located relative to the input sequence, since it determines which of (and to what extent) the input samples contribute to each output sample. These two choices are related to each other, as will be seen.

Let us assume that the input samples, $f(i)[i=0, \ldots, n-1]$, are located at integer spatial positions $x_{f}(i)$, from 0 to $n-1$. Then, the output samples, $g(j)[j=0, \ldots, n / r-1]$, will be located at spatial positions $x_{z}(j)$, such that $x_{8}(j)=j r+(r-1) / 2$. This choice yields $x_{g}(0)=(r-1) / 2$ instead of $x_{g}(0)=1 / 2$ as Burt (1981, p. 33) suggested. It makes the output sequence perfectly centered with regard to the input sequence, as shown by the bullets in Figure Al for the $r$ we are concerned with.

Depending on the particular fractional order, some output samples may wind up located at integer spatial positions (Type I samples, being exactly at the same location as a given input sample), while some others may occur at half-integer spatial positions (Type II samples, being exactly midway between the location of two consecutive input samples) or somewhere else

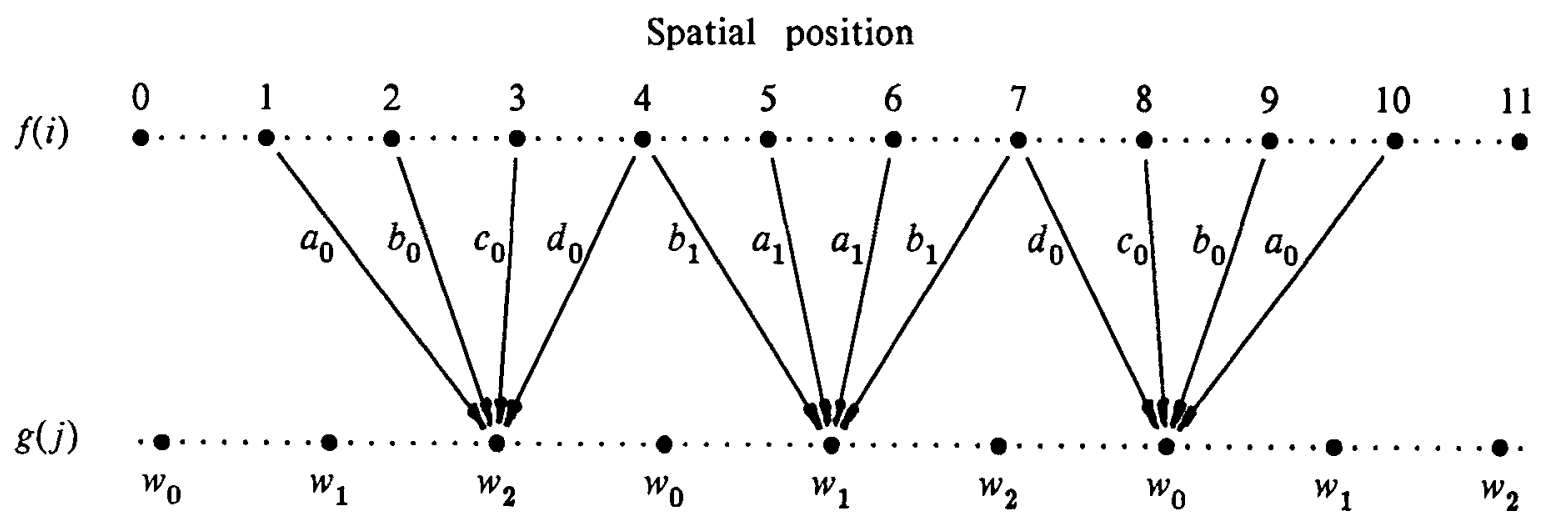

Figure A1. Fractional HDC with order $r=4 / 3$. Bullets represent sample points (at their spatial locations) for both the input (upper) and output (lower) sequences. The three generating kernels are shown as patterns of arrows connecting four input samples to the output samples that are to receive the result of their weighted average. A label below each output sample indicates which generating kernel is used to determine the output value at that location. The labels for each arrow indicate the contribution (weight) of input samples to output samples for each kernel. 
(Type III samples, being between the locations of two consecutive input samples but closer to one of them). Figure A1 shows that only Type II and Type III samples occur when $r=4 / 3$. In all cases, we let the width of the weighting function or generating kernel be four (i.e., four input pixels contribute to each output value). However, note in Figure Al that several generating kernels will be needed, since the distance relationships of output pixels to input pixels vary. In fact, there are $k_{2}$ such distance relationships, and $k_{2}$ generating kernels, $w_{t}(x)[t=0$, $\left.\ldots, k_{2}-1\right]$, are actually needed. Nonetheless, within each block of $k_{2}$ pixels, the positioning of output samples with regard to input samples is such that there are $\left(k_{2}-1\right) / 2$ pairs (for odd $k_{2}$ ) of output pixels whose distance relationships to input samples are the same, but in the opposite direction. Hence, the generating kernel to be applied to one of the pixels in this pair is the mirror reflection of that applied to the other. In this case, then, we need three generating kernels, each of which is applied to compute the value at a single output pixel within each block of size $k_{2}$. The particular kernel that is used at each output sample is shown in Figure Al under the corresponding pixel. The algorithm for this fractional HDC is as follows:

$$
\begin{aligned}
& \text { for } j=0 \text { to } n / r-3 \text {, step } 3, \\
& \qquad g(j)=\sum_{i=-1}^{2} w_{0}(i-1 / 6) f(i+r j) ;
\end{aligned}
$$

for $j=1$ to $n / r-2$, step 3 ,

$$
g(j)=\sum_{i=-1}^{2} w_{1}(i-1 / 2) f(i+r j-1 / 3)
$$

for $j=2$ to $n / r-1$, step 3 ,

$$
g(j)=\sum_{i=-1}^{2} w_{2}(i-5 / 6) f(i+r j-2 / 3) .
$$

The arrows in Figure Al exemplify these three forms of weighted averaging. Any of the boundary conditions described in García-Pérez (1988) can, among others, be applied to compute the value at the border pixels in the output sequence.

Once the output samples are positioned and the width of the generating kernels is determined, the only thing that remains is to decide which weights to use. There is not much room for choice, since the generating kernels are subjected to several constraints that determine all their weights save one (given the width we have chosen). For Type I samples (which do not exist when $r=4 / 3$ ), we apply the constraints pertaining to odd HDC generating kernels (see Burt, 1981, p. 23; see also García-Pérez, 1988, for a slight modification of these constraints). For Type II samples, we apply the constraints on even HDC generating kernels (Burt, 1981, p. 28; or García-Pérez, 1988). Finally, for Type III kernels we apply the constraints that are specific to fractional HDC (Burt, 1981, p. 34). For order $r=4 / 3$, this implies that

$$
\begin{aligned}
& w_{0}(-7 / 6)=w_{2}(7 / 6)=a_{0}=165 / 354-35 b_{0} / 59 \\
& w_{0}(-1 / 6)=w_{2}(1 / 6)=b_{0}, \text { with } 77 / 216 \leq b_{0} \leq 35 / 66 \\
& w_{0}(5 / 6)=w_{2}(-5 / 6)=c_{0}=154 / 354-13 b_{0} / 59 \\
& w_{0}(11 / 6)=w_{2}(-11 / 6)=d_{0}=35 / 354-11 b_{0} / 59 \\
& w_{1}(-1 / 2)=w_{1}(1 / 2)=a_{1}=51 / 236+24 b_{0} / 59 \\
& w_{1}(-3 / 2)=w_{1}(3 / 2)=b_{1}=67 / 236-24 b_{0} / 59
\end{aligned}
$$

Letting $b_{0}=0.39$ makes the kernels approximately Gaussian in shape. Each arrow in Figure Al is labelled with the name given to its weight in the above expressions.

Note that, in Figure A1, the interpixel distance increases from input to output. However, insofar as the sequences $f(i)$ and $g(j)$ are regarded as arrays, location information is lost, and, in fact, $g$ is a compressed version of $f$, since it represents the same picture with three fourths the pixels.

(Manuscript received September 9, 1988; accepted for publication November 30,1988 .) 